\title{
The blurring of the margins of endocrinology and its relevance to the paediatric endocrinologist
}

\author{
Ieuan Hughes \\ Department of Paediatrics, University of Cambridge, Cambridge CB2 2QQ, USA \\ (Correspondence should be addressed to I Hughes; Email: vicki.norton@endocrinology.org)
}

The word hormone was coined a century ago by Bayliss and Starling when they described the discovery of secretin $(1,2)$. Coupled with the knowledge that hormones were chemical messengers produced at one site but mediating their actions at a distal site, the specialty of endocrinology has traditionally focussed on diseases associated with endocrine glands producing either excessive or inadequate amounts of hormone. Later, it became apparent that a range of disorders were due to hormone resistance, characterised by lack of response to a hormone despite normal or usually increased circulating concentrations of the cognate hormone (3). Disorders of the mainstream endocrine glands (pituitary, thyroid and parathyroids, adrenals, gonads and pancreas) still provide the basis for the syllabus in endocrinology, but now enhanced by the emergence of other organs that also produce hormones. These include the heart, lungs, kidneys, liver, gut and brain. To this list must be added the skin (the largest organ in the body), the vascular endothelium, bone and cartilage and fat tissue.

Adipose tissue has assumed prime of place in endocrinology with the emergence of obesity as a major disease affecting a large proportion of the population. The adipocyte is a veritable factory of hormones producing adipokines such as leptin, resistin and adiponectins. How these relate to appetite control and metabolic balance is now the subject of massive research not only in basic endocrinology, but also in the pharmaceutical industry. How does the paediatric endocrinologist relate to this widening of the boundaries to endocrinology?

Paediatric endocrinology as a specialty is a relative newcomer on the endocrine scene and indeed, nurtured by a non-paediatric physician. The textbook realised by Lawson Wilkins et al. on the diagnosis and management of treatment of endocrine disorders in childhood and adolescence (4) remains a classic of clinical descriptions even if it is long outdated on the biochemical aspects of the subject. The study of growth and puberty has always signalled out the paediatrician from the adult

Proceedings of the 4th Ferring Pharmaceuticals International Paediatric Endocrinology Symposium, Paris (2006). Ferring Pharmaceuticals has supported the publication of these proceedings. endocrinologist. Since growth is generally an indicator of health and well being in infants and children, the clinical practice of the paediatric endocrinologist is locked in to mainstream paediatrics. Seldom does the paediatric endocrinologist 'stray' into the territory of the adult endocrinologist for clinical practice or vice versa. That must be so in view of the different skills and training needed to practice at different points of the age span. The adult physician would not be expected to manage the infant with ambiguous genitalia of the newborn any more than the paediatrician would be expected to grapple with a complex case of acromegaly. Nevertheless, the boundaries of endocrinology are now becoming so blurred that it is incumbent on endocrinologists of whatever ilk to at least be aware of the mushrooming breadth of the subject and consider facets that may be relevant to individual clinical practice.

Take, for example, the concept of programmed events activated in foetal life or in early infancy and then only manifest in some measurable format in adult life. The phenomenon now considered under the umbrella title of 'developmental origins of adult disease' (5) transcends virtually the whole of clinical medicine. Its origin is within the purview of the paediatric endocrinologist, who is knowledgeable about the causes of foetal growth restraint, its consequences at birth and early infancy, whether it is followed by catch-up growth or not, and how this may affect puberty thereafter. Yet, it is still not possible to determine precisely what triggers puberty in humans at around 10-11 years of age. Each review of the subject over the past 20 years has made some headway in completing the jigsaw, the most recent adding the GPR54 kisspeptin receptor and environmental chemicals to the multi-component model of the control of the onset of puberty (6). The paediatric endocrinologist in clinical practice is not party to the adult consequences of foetal growth restraint as manifested by the metabolic syndrome and subsequent cardiovascular disease. Indeed, the associations with foetal growth stretch beyond the classic metabolic syndrome to include reproductive abnormalities, some cancers and certain psychiatric diseases. This broadening of the horizon by viewing with a wide-angle lens is the concept developed in the programming for the 'Fourth Ferring Pharmaceuticals Paediatric Endocrinology Symposium' and recorded in print in this 
special issue of the European Journal of Endocrinology, also supported by Ferring.

Traditional paediatric endocrinology is not the subject of this edition, yet all the topics impinge on the intellectual framework within which the paediatric endocrinologist practises his or her art and science. Short stature and puberty are the raison d'être for the paediatric endocrinologist, but in this instance, it is the margins of normal short stature as a 'disease' or not which is considered, rather than from the perspective of classic endocrine gland deficiency coupled to hormone replacement. There can be no more powerful example of programming than the evidence of sex dimorphic behaviour in humans attributed to prenatal exposure to androgens. Who would have thought that the paediatric endocrinologist should know the origins of autistic spectrum disorder were possibly hormonally determined in foetal life? This special edition is packed with interesting facts, such as evidence that breast development appears to be starting earlier but menarchal age is not changing; is more harm done to the majority of normal short children by routine growth measurements? the metabolic syndrome affects $16 \%$ of adults above age 20 years; males have a 'timid' gene, which is passed on by their mothers; there is sex dimorphism in brain structure, but the similarities are more evident than the differences; androgens do not enhance intellect, but assertive mothers have assertive daughters; the criteria for gender identity disorders in adolescents are well validated for which there is a clinical management strategy that allows the adolescent to explore the options of sex reassignment prior to any definitive surgical procedures.

The spectrum of topics covered in this special edition give a flavour of the breadth of knowledge the paediatric endocrinologist has to be aware of beyond that required for the routine clinical practice of the subject. This is the case for the present; what of the future? Endocrinology, like all medical specialties, will change its boundaries of practice consequent upon the biomedical revolution manifest in genomics, transcriptomics, proteomics and metabonomics. Recent predictions have been made as to what endocrinology will look like towards the latter part of this century (7-9). The impact of obesity sits high on the agenda, but it is the paediatric physician who has to grasp the nettle from the perspective of prevention (10). Pari passu, there will be a huge investment in drugs targeted at the hormones and receptors that regulate energy homeostasis. Hormone replacement will become more refined with improved delivery devices, longer-acting hormone formulations and the clinical application of pharmacogenomics. The boundaries of endocrine, paracrine, intracrine and neurocrine signalling are already recognisably blurring, as exemplified by the actions of growth factors and tissue-specific regulation of enzyme modulators of hormone synthesis and metabolism. Even cosmetic endocrinology has to be added to the futuristic list in relating to enhancing growth in short children, curing baldness or unwanted hair, improving sexual function and general well being. The emphasis is on aging, yet it is perhaps at the beginning of life that the mechanisms relevant to this degenerative process are programmed and need to be studied.

The practising paediatric endocrinologist will continue to occupy the bulk of the working day in the management of short stature, problems of puberty, thyroid disorders, genital abnormalities and, increasingly, obesity-related problems. It can be refreshing to stand back occasionally from such pressing clinical needs and consider the wider framework that endocrinology now embodies and is relevant to the paediatrician. A sample of that process is contained in this special edition.

\section{References}

1 Bayliss WM \& Starling EH. The mechanism of pancreatic secretion. Journal of Physiology 190228 325-353.

2 Starling EH. The chemical control of the functions of the body. Lancet 19052 339-341. 423-425, 501-503, 578-583.

3 Hormone Resistance Syndromes, pp 281. Ed JL Jameson. New Jersey: Humana Press, 1999.

4 The Diagnosis and Treatment of Endocrine Disorders in Childhood and Adolescence, edn 3, pp 619. Eds L Wilkins, RM Blizzard \& CJ Migeon. Springfield, Illinois: Charles C Thomas, 1996.

5 Gluckman PD, Hanson MA \& Pinal C. The developmental origins of adult disease. Maternal and Child Nutrition 20051 130-141.

6 Aubert ML \& Pralong FP. Puberty: a sensor of genetic and environmental interactions throughout development Molecular and Cellular Endocrinology 2006 254-255 1-233.

7 Ho KKY. Endocrinology: the next 60 years. Journal of Endocrinology $20061903-6$.

8 Dhillo WS, Murphy KG \& Bloom S. Endocrinology: the next 60 years. Journal of Endocrinology $2006 \mathbf{1 9 0} 7-10$.

9 Waters MJ. Endocrinology: the next 60 years - the helix and the chip. Journal of Endocrinology $200619011-12$.

10 Ong KK \& Loos RJ. Rapid infancy weight gain and subsequent obesity: systematic reviews and hopeful suggestions. Acta Paediatrica $200695904-908$. 